\title{
SENDEROS DE LA GEOGRAFÍA CULTURAL
}

\section{Adrián Hernández Cordero* \\ Universidad Autónoma Metropolitana}

\section{CAMINHOS DA GEOGRAFIA CULTURAL}

Resumo: O presente artigo pretende reflexionar sobre as trajetórias da geografia cultural, um ramo da Geografia Humana que surgiu no início do século XX e tem sido muito importante, especialmente no mundo anglo-saxão. Dentro desse contexto, refletimos sobre a concepção de cultura desenvolvida em vários momentos, passando de uma abordagem materialista e estática a uma abordagem intangível e dinâmica. Desta forma, uma mudança de direção foi gerada na maneira de entender a relação entre espaço e sociedade.

Palavras-chave: Geografia humana, espaço, cultura, teoria social.

\section{TRAILS OF CULTURAL GEOGRAPHY}

Abstract: The aim of the present article is to analyse the trajectories of the Cultural Geography. It is a recent branch of Human Geography which started to develop in the beginnings of XX century and had gained a lot of popularity since then, especially in the Anglo-Saxon context. The paper reviews the diachronic development of the concept of culture in the field of the Cultural Geography. The document highlights the transition from a materialist and static approach to a dynamic and intangible one which has caused a transformation in the way of understanding the relation between space and society.

Keywords: standard-years classification; standard-months classification; rainy days; rainfall variability.

Resumen: El artículo tiene como finalidad reflexionar sobre las trayectorias de la geografía cultural. Una rama joven de la Geografía Humana, que surgió a principios del siglo XX y que ha tenido un encumbramiento en la disciplina, sobre todo en el ámbito anglosajón. En este sentido, se reflexiona sobre la concepción de la cultura que ha desarrollado la geografía cultural en diversos momentos, transitando de un enfoque materialista y estático hacia otro intangible y dinámico. De este modo, se generó un cambio de dirección en la forma de comprender la relación espacio y sociedad.

Palabras-clave: Geografía humana, espacio, cultura, teoría social. 
Introducción

La relación entre espacio y cultura es uno de los campos más fértiles para la geografía. En los últimos años este tópico ha generado amplios debates teóricos y metodológicos en las naciones que gozan de tradición en los estudios socio territoriales y que se mantienen como punteras en la producción científica; concretamente se pueden mencionar a Reino Unido, Estados Unidos y Francia.

La cultura fue redescubierta por los geógrafos a partir de los años ochenta del siglo pasado. Ello ocurrió en un contexto de veloces y profundos cambios a escala planetaria en el que se cuestionaron los paradigmas modernos. MINCA (2002) argumenta que las teorías y explicaciones que sustentaron el mundo fueron derribadas, tanto como el propio Muro de Berlín que marcó el fin de una época. Se rompió con la visión hegemónica de Occidente y progresivamente se aceptaron e incluso encumbraron miradas diferentes que por mucho tiempo fueron ignoradas. La alteridad y los grupos subalternos tomaron protagonismo en las agendas de investigación de las ciencias sociales y las humanidades.

Ante este panorama, los científicos sociales tuvieron que replantearse sus explicaciones, acrecentar sus cajas de herramientas metodológicas, así como cuestionarse su propia labor en el mundo. LINDÓN (2010) argumenta que la geografía no escapó a este respecto y logró romper con su enfoque tradicional y rígido. Paulatinamente las fronteras académicas que durante mucho tiempo estuvieron resguardadas se fueron haciendo difusas. La geografía incrementó sus lazos con otras disciplinas, ya no únicamente hubo dialogo con sus compañeras centenarias, la historia y las ciencias naturales, sino que desde ese momento abrazó a la sociología, la lingüística, la psicología social, la antropología, etcétera. La geografía se abrió y, para usar las palabras de CLAVAL (1999), aprendió a estar de lado de las ciencias sociales.

En este diálogo interdisciplinario, la geografía se reorganizó a partir del giro cultural que permeó al conjunto de las disciplinas académicas. A partir de ello, la ciencia geográfica renovó la concepción de cultura que utilizó afanosamente por mucho tiempo. Paralelamente, ocurrió un giro espacial mediante el cual la geografía y su objeto de estudio, el espacio (a través de diferentes categorías como territorio, región, paisaje, lugar) fue redescubiertos por las ciencias sociales, quienes al fin comprendieron que las personas no están suspendidas en el aire como sostuvo Ratzel desde 1898. De esta manera, la espacialidad y la cultura comenzaron un camino conjunto que ha llevado a fértiles discusiones.
Es en este contexto, el presente escrito tiene como objetivo general reflexionar sobre el vínculo entre espacio y cultura, a partir de efectuar un sintético recorrido por la geografía cultural. El ensayo consta de seis apartados. En primer lugar, se plantean una serie de coordenadas que ayudan a situar los antecedentes del estudio de la cultura en la geografía. Concretamente, se revisan dos de los grandes padres de la geografía moderna: Friedrich Ratzel y Paul Vidal de La Blache. Posteriormente, se aborda la figura del geógrafo estadounidense Carl Sauer, fundador de la geografía cultural clásica que operó bajo una noción de cultura objetiva, homogénea y súper orgánica. En un tercer momento, se plantea la forma en que los postulados de la geografía cultural estadounidense entran en crisis durante la segunda mitad del siglo pasado. Sobre todo, por la coyuntura de la Posguerra que planteó una serie de demandas específicas y pragmáticas a la geografía que la Escuela de Berkeley era incapaz de asumir. Consecutivamente, se analiza el surgimiento de la denominada nueva geografía cultural hace cerca de tres décadas en el Reino Unido. Esta corriente de pensamiento fue rupturista y criticó la forma en que se venía trabajando lo cultural desde el siglo XVIII en la geografía. A partir del giro cultural se interesó en la dimensión inmaterial de la cultura, abriendo múltiples posibilidades de estudio. En quinto lugar, se ofrece una tabla que contrasta a la geografía cultural tradicional y la renovada geografía cultural. Su finalidad es hacer patente las diferencias que se expondrán a lo largo del texto. Asimismo, se presentan un par de esquemas de la forma en que ambas escuelas de pensamiento conciben la cultura. Las comparaciones indagan la relación entre cultura y espacialidad. La geografía saueriana se apoyó en una visión de espacio contenedor que marchó hacia una suerte de determinismo ambiental y cultural. Mientras que la nueva geografía cultural recurrió a una visión del espacio social y constructivista, en la cual se reconoce la autonomía de los sujetos y su interacción con el espacio. Se concluye con una serie de reflexiones finales y posibles vetas de investigación.

Los antecedentes de la relación entre geografía y cultura

Entre los siglos XVIII y XIX en Europa se vivieron una serie de sucesos que la transformaron. Entre éstos se encuentra la irrupción de la llustración que trastocó profundamente la esfera científica, política, técnica y cotidiana. Con el Siglo de las Luces se rompió con los atavismos feudales y comenzó una nueva etapa que 
buscaba la desacralización de la realidad. Las maneras de comprender al mundo fueron revolucionadas a partir de los postulados racionalistas que impactaron en los grandes pensadores de la época. Éstos adoptaron el método científico deductivo que buscaba ofrecer explicaciones objetivas a los fenómenos terrestres.

La geografía no fue ajena a la metamorfosis: a mediados del siglo XIX se marcó una ruptura con la forma en la que se había entendido y desarrollado. Según ORTEGA VALCÁRCEL (2000), las nuevas ideas plantearon el surgimiento de una disciplina que superó la vieja representación del mundo, pero que sobre todo introdujo elementos científicos (observables, medibles y refutables) al quehacer geográfico. Asimismo, se acompañó de la progresiva conformación de una comunidad geográfica visible en círculos académicos y políticos. Todo ello representó el punto de partida de lo que se conoce como la geografía moderna.

Entre la nueva generación de geógrafos, principalmente alemanes, destaca la figura de Friedrich Ratzel, quien a partir de los preceptos naturalistas y evolucionistas de Darwin estudió la forma en que el medio ambiente imponía sus condiciones sobre los grupos humanos. Así, se creó la corriente del determinismo geográfico. En este marco, Ratzel propuso por primera ocasión el término de geografía cultural, que sirvió para diferenciarse de la geografía física, así como para explicar la distribución de las diferentes culturas en la superficie terrestre y su movilidad en el territorio (FERNÁNDEZ CHRISTLIEB, 2006; CRANG, 1998). Debe mencionarse que el término cultura desde la óptica ratzeliana se entendió como sinónimo de civilización. Según está visión, el grado de desarrollo de los pueblos influía en la forma en que se adaptan al medio. Los grupos humanos con mejores condiciones afrontaron de forma óptima los desafíos naturales, mientras que ocurrió lo contrario con aquellos que contaban con menores aptitudes. Por lo tanto, la cultura sirvió para justificar la existencia de pueblos más capaces que se impondrán sobre los menos hábiles.

En la historia del pensamiento geográfico el principal antagonista de los postulados de Ratzel es Paul Vidal de La Blache, debido a que el líder de la escuela de geografía francófona trazó una alternativa a las ideas deterministas ratzelianas. En palabras de CLAVAL (1974), Vidal estableció su distancia respecto al ambientalismo para demostrar que las relaciones entre las personas y el medio eran complejas, por lo que resultaba imposible explicarlas de manera unidireccional. Vidal sentó las bases del posibilismo, argumentando que los grupos humanos contaban con la capacidad de transformar el medio según sus necesidades, a partir de los conocimientos y técnicas de las que disponían. La obra de Vidal buscaba explicar las relaciones entre sociedades y medio ambiente, poniendo en el centro de sus explicaciones el tema de la cultura (CLAVAL, 2003).

Para Vidal, el concepto clave para lograr confrontar a la naturaleza es el género de vida. Éste se puede entender como el conjunto de instrumentos (técnicas) con los que cuentan las sociedades para adaptarse y modificar el territorio. Para la escuela vidaliana los géneros de vida fueron la máxima expresión de la cultura, entendiéndose como "todo aquello que se interpone entre el hombre y el ambiente, todo aquello que humaniza el paisaje" (GIMÉNEZ, 2007:168).

La definición de cultura posibilista y la manera de estudiarla fue más sólida que la planteada por Ratzel. El abordaje cultural de la geografía francesa básicamente se enfoca en la capacidad transformadora de las personas sobre el espacio. Para BERDOULAY (2012) esto es posible a partir de los vínculos que establece un grupo humano mediante sus tradiciones y costumbres, con la intención de perpetuarse en un espacio bien definido. La acción humana produce paisajes y éstos a su vez conforman regiones; entendidas estas últimas como los espacios de escala intermedia (entre lo nacional y lo local) definidas a partir de la personalidad que les imprimen los grupos humanos. Evidentemente, estamos ante el surgimiento de lo que posteriormente se denominará regiones culturales.

\section{Carl Sauer y la geografía cultural}

El trabajo seminal de Vidal de La Blache fue continuado por sus discípulos (Febvre, De Martonne, Sorre), colocando en el foco de sus análisis el estudio de espacios tradicionales y/o agrarios. Su influencia fue notable tanto en Europa como en el resto del mundo, incluido México.

La estafeta de los estudios entre espacio y cultura salió del continente europeo y la tomó, durante la segunda década del siglo XX, el geógrafo estadounidense Carl Sauer, fundador de la Escuela de Berkeley. Él al igual que varios de los grandes maestros de la geografía moderna (Humboldt, Ritter, Ratzel) contó en un primer momento con una sólida formación en geografía física, concretamente en geología. Además, Sauer provenía de una familia de inmigrantes alemanes, por lo cual tuvo contacto con las aportaciones de la ciencia geográfica de aquel país, influenciada por el estudio de la naturaleza. A partir de estas situaciones, la obra de Sauer está marcada por retomar algunos elementos del estudio de la formación de los suelos para examinar a las socie- 
dades y su relación con el territorio a través del paisaje.

El paisaje en la obra de Sauer es el concepto medular de la geografía. Éste es entendido como el conjunto de formas creadas por la acción humana sobre la naturaleza (LOBATO, 2001), los cuales evidencian elementos tanto de origen natural como cultural (FERNÁNDEZ CHRISTLIEB, 2006). El paisaje puede pensarse como la síntesis objetiva de la relación sociedad-medio, que es posible gracias a la cultura. De nueva cuenta, se está ante el planteamiento de que la cultura es el instrumento que configura el territorio. Cabe recordar la célebre frase de SAUER que clarifica esta idea: "La cultura es el agente, el área natural es el medio, el paisaje cultural es el resultado" (1925).

El estudio del paisaje se llevó a cabo a partir de la comprensión de sus dinámicas internas, con la finalidad de ejecutar la diferenciación espacial. Ello se debe, según FERNÁNDEZ CHRISTLIEB (2006), a que Sauer contaba con una visión de la geografía como una disciplina corológica. La diferenciación de la superficie terrestre se contraponía al enfoque positivista que imperaba en aquel momento y que buscaba la creación de leyes generales. La tendencia de la geografía cultural estadounidense de estudiar las particularidades físicas y étnicas a través de áreas culturales tuvo un buen maridaje con la teoría antropológica contemporánea del relativismo cultural ${ }^{7}$. De esta manera, se ponía atención en la diversidad, en las particularidades locales y en la materialización de los rasgos culturales de comunidades tradicionales en territorios bien definidos.

El examen del paisaje desde una postura cultural no sólo contaba con una perspectiva sincrónica, sino que también se caracterizó por estudiar su dimensión diacrónica. LOBATO (2001) señala que Sauer y sus discípulos se interesaron en estudiar las huellas de los grupos humanos en el paisaje. Concretamente en cómo las comunidades a partir su actividad cotidiana dejaban marcas que debían ser interpretadas a través de la cultura. En síntesis, siguiendo a LUNA (1999), el objetivo de la escuela saueriana fue la reconstrucción histórica del paisaje, a partir de la fuerza de elementos culturales materiales (las características de las viviendas o las técnicas de cultivo) e inmateriales (el uso de lenguas maternas).

\section{El declive de la geografía cultural}

La geografía cultural de la Escuela de Berkeley se mantuvo como la corriente más productiva e importante del globo hasta cerca de la segunda mitad del siglo

'En términos generales el relativismo cultural impulsado por el antropólogo Franz Boas manifiesta que no existe una cultura universal, sino que el mundo se conforma por culturas particulares. En este sentido, las culturas deben ser entendidas en su contexto.
XX, gracias al liderazgo académico de Sauer, a su producción científica y su formación docente. Al respecto, LUNA (1999), menciona que el padre de la geografía cultural dirigió, durante tres décadas, más de 40 tesis de doctorado de geógrafos estadounidenses, quienes se encargaron de difundir sus ideas por diversas latitudes.

La jubilación de Sauer conllevó a que la geografía cultural perdiera parte de su impulso. Sin embargo, el declive de ésta tiene más explicaciones. Destaca la emergencia de nuevos paradigmas como la geografía neopositivista o teorética cuantitativa, impulsada por Fred Schaefer. Éste confrontó el enfoque ideográfico del que se nutrió la geografía cultural, ya que consideraba, en palabras de CAPEL (1981), que carecía de cientificidad al estudiar las particularidades de la superficie terrestre y no generar leyes, así como también por recurrir a la descripción y no a la explicación.

En el contexto de la Posguerra y el papel hegemónico que desempeñó Estado Unidos en la reconstrucción de Europa se requerían enfoques pragmáticos como el de los geógrafos neopositivistas. Ellos a través de modelos matemáticos pusieron su conocimiento al servicio de políticas de ordenación territorial. En este sentido, según ORTEGA VALCÁRCEL (2000), la geografía comenzó a tratar la organización del espacio y progresivamente se olvidó de conceptos como paisaje y medio que para entonces sonaban vetustos.

Durante la década de los años sesenta se vivió un cisma político a escala planetaria abanderado por el avance del comunismo (a través de la Revolución Cubana) en el entonces denominado Tercer Mundo. Mientras que en países industrializados tuvieron auge múltiples movimientos sociales que recogían reivindicaciones pacifistas y por los derechos civiles; por ejemplo, los sectores afrodescendientes y feministas. Este marco no fue ajeno a la geografía y a partir del redescubrimiento del marxismo se comenzó a gestar una nueva corriente denominada geografía radical. Ésta reprobó el servilismo de la geografía teorética, así como la visión anacrónica y apolítica del enfoque ideográfico. Su aporte se fundamenta en el compromiso social de la disciplina para contribuir a paliar las desigualdades propias del sistema capitalista (GÓMEZ MENDOZA ET AL., 1982).

Las nuevas discusiones en torno a la geografía ocasionaron que el estudio de la cultura fuera prácticamente borrado de los debates que se estaban originando. Se consideraba que la forma en que se venía practicando la geografía cultural, así como sus temas de estudio se encontraban desfasados ante las interesantes y apabullantes modificaciones que estaban ocurriendo a nivel planetario. Sin lugar a dudas, la geografía cultural se había quedado obsoleta para una realidad en constante transformación, 
sobre todo a partir de la segunda mitad del siglo pasado.

\section{La nueva geografía cultural}

Los cambios ocurridos en el mundo y en la geografía implicaron que la atención se pusiera en nuevos paradigmas como las geografías radicales y humanistas enfocadas en temas emergentes para la disciplina. Mientras que los tópicos culturales pasaron por un periodo de hibernación. Fueron redescubiertos cuando ya que se perfilaba el cambio de centuria, concretamente en los años ochenta, reflejando las modificaciones que se asocian con la desintegración de la Unión Soviética, el derrumbe de la utopía socialista, el triunfo del sistema capitalista y la adopción del modelo neoliberal a escala planetaria (KRAMSCH, 1998). Paralelamente se desencadenaron, según ZUSMAN y HAESBAERT (2011), un conjunto de factores concomitantes como la mercantilización de diversas expresiones culturales, la homogeneización de modas, valores y creencias a partir de la globalización, y el incremento de la movilidad de la población por fenómenos como la migración y el turismo.

Dichos cambios, marcados por la velocidad y la profundidad, han implicado un parteaguas en el ámbito de las ciencias sociales, ya que debían intentar ofrecer respuestas a las múltiples preguntas germinadas en terrenos teóricos y metodológicos. De esta manera, surgen los denominados giros ${ }^{2}$ que buscan brindar nuevos discursos para estudiar la realidad. En el caso de la geografía, los giros generaron el viraje de dirección en la manera de estudiar la relación espacio-sociedad. Según (LINDÓN, 2010). cuestionaron la forma de producción de conocimiento geográfico a través de poner en tela de juicio los conceptos, plantearon nuevas o diferentes temáticas de estudio y trazaron una revisión de las estrategias metodológicas.

Entre los diversos giros que existen uno de los que ha tenido mayor repercusión en la geografía ha sido el giro cultural. JACKSON (1999) arguye que éste se puede entender por el cambio de rumbo de la geografía que incorpora nuevas maneras de estudiar lo cultural, a partir de distintas formas de análisis del discurso, así como la incorporación de los tópicos de la representación y la diferencia. Asimismo, el giro cultural se puede entender como "el desplazamiento intelectual que ha situado a las variables culturales en el centro de los debates contemporáneos (al mismo nivel que las variables económicas y políticas)" (NOGUÉ y ALBET, 2004: 159). La cultura deja de tener un papel secundario y se asume

${ }^{2}$ Se debe dejar constancia de que existe una variedad de giros (lingüístico, biográfico, pictórico, espacial, etc.), aunque en este trabajo por su naturaleza no se abordarán, pero es importante mencionarlos. como protagonista de las transformaciones sociales.

CLAVAL (2010) sostiene que el giro cultural obliga a repensar la cultura y a reconstruirla. En este ejercicio reconstitutivo o deconstructivo para usar los términos derridianos se generaron una batería de críticas a los postulados de la geografía cultural clásica, así como al propio concepto de cultura. Sobre todo, a las ideas manejadas por la Escuela de Berkeley, las cuales fueron las preponderantes durante varias décadas del siglo XX. Entre los reproches se hallan principalmente:

\begin{abstract}
El reduccionismo material. La geografía cultural se interesó en los aspectos materiales de la cultura, analizando las formas físicas que eran evidentes para el observador. Con lo que se omitieron las dinámicas sociales que subyacen a la producción del modelado de la superficie terrestre (CRANG, 1998; LINDÓN, 2010; LUNA, 1999).

Determinismo cultural. Los geógrafos culturales usaron el concepto de cultura de manera tal que era considerada como una entidad súper orgánica. En otras palabras, la cultura era externa a la sociedad e ignoraba el papel de los sujetos, los cuales son vistos únicamente como difusores de ésta. La cultura tenía tanta fuerza y propósitos propios que se impone sobre los pueblos. De esta manera, se generó un determinismo cultural en el cual el espacio era un mero contenedor de la cultura (LUNA, 1999; LINDÓN, 2016)

El sesgo histórico. La geografía teorética-cuantitativa consideró que la geografía cultural estaba ensimismada en unos intereses academicistas volcados sobre el pasado. Mientras que el presente no les interesaba $y$ por lo tanto, pensaban a la cultura como un ente estático, dejando a un lado temas de singular relevancia para el crecimiento económico (LOBATO, 2001; KRAMSCH, 1999).

La instrumentalización política. La geografía radical denunció que los estudios que realizaba la geografía cultural norteamericana en países latinoamericanos, africanos y asiáticos estaban auspiciados por el gobierno estadounidense. Bajo el argumento de llevar a cabo comparaciones culturales sirvieron para alimentar órganos de inteligencia anti revolucionaria (LOBATO, 2001).
\end{abstract}

Los puntos anteriores muestran que con el giro cultural se dinamitaron los cimientos del edificio teórico y metodológico de la cultura, que se habían mantenido en el pensamiento geográfico durante un periodo significativo.

El péndulo de las vanguardias geográficas se ubicó de nueva cuenta en Europa. En el Reino Unido ${ }^{3}$ un conjunto de geógrafos, influenciados por la Escuela

${ }^{3}$ En este trabajo se habla de la nueva geografía cultural desde una perspectiva global, aunque no se debe dejar de mencionar que ésta también tuvo eco en Francia e incluso mantiene una serie de divergencias respecto a los trabajos ingleses. Sin embargo, la nueva geografía cultural anglosajona ha sido la que ha acaparado mayores reflectores en los debates actuales. Para mayores referencias de la nueva geografía cultural en su versión francesa pueden revisarse los trabajos de DI MÉO (2008), COLLIGNON (1999) y CLAVAL (1999). 
de Birmingham ${ }^{4}$, conformaron una corriente de pensamiento denominada como la nueva geografía cultural. En un principio sus máximos exponentes fueron Peter Jackson y Denis Cosgrove, quienes plantearon que se debían abrir veredas para estudiar aspectos desatendidos, ignorados e incluso despreciados por la geografía como son las categorías de género, sexualidad, identidad o etnia (JACKSON 1980; COSGROVE, 1983). Éstas debían de ser estudiadas en su relación con diferentes nociones espaciales (lugar, región, paisaje, territorio), las cuales también estaban siendo revisitadas.

En la nueva geografía cultural existió un golpe de timón respecto a la forma en que se comprendía la cultura. Dejó de ser pensada como un ente externo a las personas, que implica un tipo de determinismo. KRAMSCH (1999) sostiene que para los nuevos trabajos geográficos, la cultura asume un papel activo y está inserta en una amplia y compleja trama de significados que repercute en la vida cotidiana.

La cultura ya no es concebida como un objeto estático que se mantenía a través del paso del tiempo, sobre todo en sociedades campesinas y/o tradicionales. Por el contrario, se adopta una perspectiva dinámica y contemporánea que pone su énfasis explicativo en "las relaciones complejas de dominación, oposición y reapropiación que caracterizan a las subculturas minoritarias" (KRAMSCH, 1999: 55), en sociedades urbanas contemporáneas. Así, el espacio de interés de la geografía cultural cambió a partir del intenso proceso de urbanización que experimentó el mundo, el cual además de implicar una alta densidad de población trajo consigo transformaciones en las formas en las que se vivían y practicaban las urbes.

Otra veta que abre la nueva geografía cultural respecto a la forma de comprender la cultura es el tránsito de lo material a lo inmaterial. Ya se dejó constancia que en la geografía moderna, gracias a los postulados positivistas, primó la idea de estudiar a la cultura desde un sesgo materialista y, por ende, objetivo. Todo aquello que fuera visible sobre el territorio era considerado como elemento de estudio para la geografía. Sin embargo, a partir de hace cuatro décadas lo inmaterial tiene cabida en la ciencia del espacio. La comunidad geográfica traspasó fronteras académicas y estudiar temas diversos como por ejemplo la forma en que las palabras construyen la realidad geográfica, es decir, "el espacio no sólo es objeto de manufactura y modelado material, sino que su constitución pone en juego pro-

${ }^{4}$ Tuvo su origen en el Centre for Contemporary Cultural Studies durante la posguerra y fue liderada por Richard Hoggart, Raymond Williams, Edward Thompson y Stuart Hall. Grosso modo estudian la cultura a través de las clases sociales y su relación con los medios de comunicación masivos, el lenguaje, la ideología, el género, por mencionar algunos. cesos más complejos que integran lo inmaterial, es decir, palabras, imágenes, fantasías" (LINDÓN, 2010: 32).

La cultura también dejó de interpretarse a partir de conjuntos humanos extensos y de ámbitos espaciales como las regiones o áreas culturales. El movimiento renovador abrió el abanico de posibilidades. Una de sus vertientes apuntó al estudio del sujeto o el individuo y su vinculación con los espacios cotidianos, en otros términos, los espacios domésticos, así como el barrio, la calle. FERNÁNDEZ CHRISTLIEB (2006) arguye que existió un cambio de escala, mientras que LINDÓN (2010) habla de un giro hacia el sujeto. Lo más relevante más allá de cómo se nombre este fenómeno es que en la actualidad se permite poner la lente en los pequeños detalles, en la vida cotidiana en relación a su contexto. Ello ha traído el rechazo a la tentación que imperaba en la geografía de buscar explicaciones y formular meta teorías que le restaban autonomía y capacidad creativa a quienes hacen este mundo: las personas.

En este contexto, también se abrió la posibilidad de explorar otros espacios relevantes, surgiendo así las geografías encarnadas (PHILO, 1999; LINDÓN, 2016) que grosso modo estudian al cuerpo como un lugar que es apropiado, significado a partir de su ensamblaje con los afectos y los sentidos.

\section{Geografías, espacios y culturas}

A lo largo de este trabajo se ha mostrado la ruptura entre la geografía cultural de vertiente clásica y la nueva geografía cultural. A pesar de que puedan existir algunos puntos de encuentro, sobre todo se hallan disonancias entre ambos paradigmas como puede verse en la Tabla 1. Ésta discute sus principales diferencias. Es pertinente señalar que se atiende la advertencia realizada por JACKSON (1999) respecto la geografía cultural en no reducir el debate a una batalla dicotómica entre lo nuevo y lo viejo.

En la tabla se puede apreciar que existe una ruptura entre ambos paradigmas, debido a que la manera en que estudian la realidad es sustancialmente diferente. Comenzando por los espacios que analizan, pasando por los sujetos estudiados, las escalas y terminando con los conceptos esgrimidos. Las razones que originan estas desigual dades ya fueron explicadas en los párrafos anteriores. En este sentido, la geografía cultural cambió y también se transfiguró la forma en que se concebía a la cultura y ello se puede observar en los siguientes esquemas. 
Tabela 1

\begin{tabular}{|c|c|c|}
\hline Características & Clásica geografía cultural & Nueva geografía cultural \\
\hline Espacio de estudio & Rural & Grupos y sujetos urbanos \\
\hline Sujetos de estudio & Comunidades tradicionales & Local-micro \\
\hline Escala de análisis & Regional & Lugar \\
\hline Conceptos usados & Paisaje y región & \\
\hline
\end{tabular}

Fonte: Elaboración propia

Esquema 1 y 2

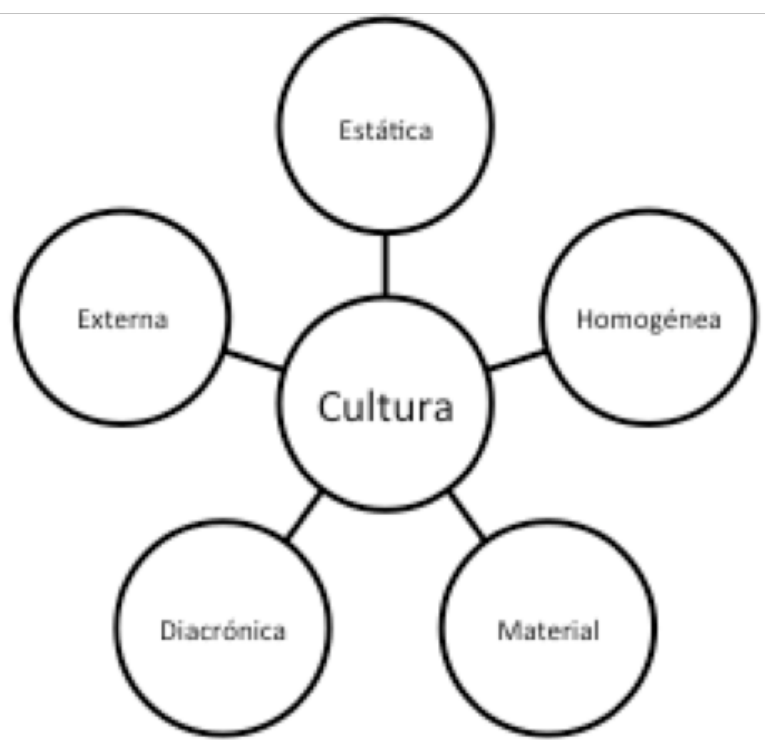

La cultura en la Clásica Geografía Cultural

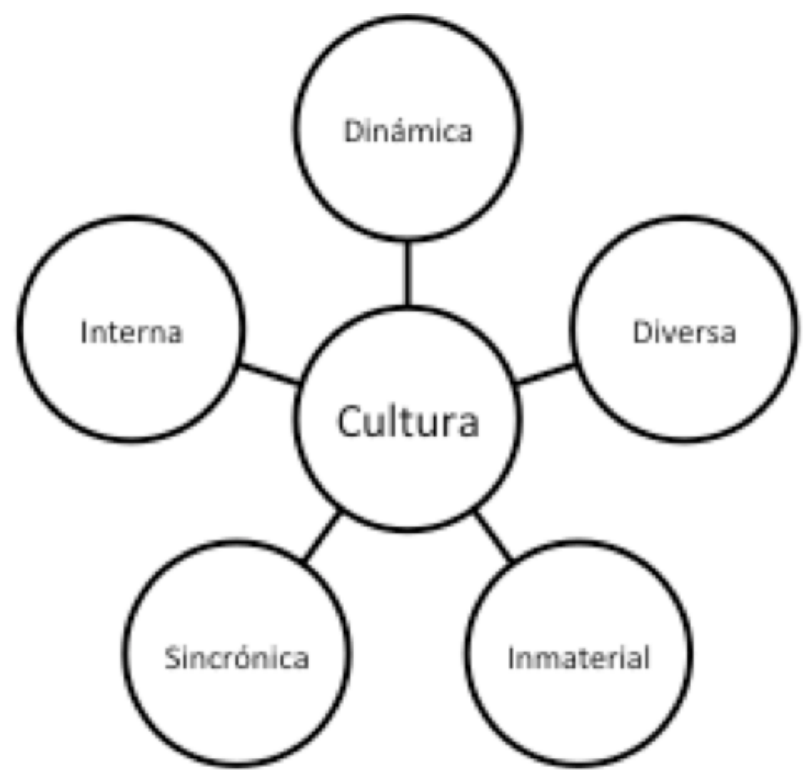

La cultura en la Nueva Geografía Cultural 
La geografía cultural de corte clásico trabajó sobre un concepto de cultura material, es decir, se interesó en el conjunto de actividades humanas que dejan su marca sobre el terreno. Mientras que la nueva geografía cultural ya no se preocupó por las formas y se centró sobre los elementos intangibles de la cultura como las palabras o las representaciones, por mencionar algunos. Al estudiar este tipo de aspectos en las urbes la nueva geografía cultural recurrió a una temporalidad sincrónica. Por su parte, la clásica geografía cultural pensaba que en las huellas había un conjunto de elementos heredados que se yuxtaponen en capas, por lo que había que recurrir a una dimensión diacrónica. Por lo tanto, la cultura se entendía de forma estática porque sus cambios se vislumbraban a través de procesos de larga duración. Cuestión contraria para la geografía cultural de los años ochenta, pues para sus seguidores la cultura se pensaba de manera dinámica y cambiante. Básicamente por el tipo de estudios que realizaba; por ejemplo, sobre las subculturas urbanas. De esta manera, la cultura se entiende como diversa, no podía ser de otra manera en las ciudades. En cambio, su predecesora teorizaba que la cultura era única y homogénea en las áreas culturales o regiones que examinaba en el espacio rural. La cultura también se entendía como una estructura potente y externa a los grupos humanos, que con su fuerza se imponía sobre la voluntad de los pueblos. En tanto, la nueva geografía cultural escapa del estructuralismo y reconoce la libertad y autonomía de los individuos.

Los elementos anteriores muestran la forma de abordar la cultura por parte de la clásica y nueva geografía cultural $y$, por ende, coexisten dos visiones distintas de espacio. Respecto a la primera, a pesar de que los principales pensadores que se revisaron en este trabajo pugnaron por reconocer la relación entre la sociedad y el medio, la cultura se miraba desde el cristal del espacio absoluto, el cual servía únicamente como escenario y soporte de las dinámicas socioculturales. En cuanto a la segunda, se observa que gracias al peso de las influencias fenomenológicas y constructivistas se otorga centralidad a los sujetos y a la forma de fundar mundos y espacios de forma dialéctica. Así, se puede hablar de la constitución de un espacio social en el que tienen un papel fundamental los espacios vividos.

La recuperación del espacio vivido que fue introducido por la geografía humanista en la década de los años setenta del siglo pasado, así como la exploración de nuevos temas de investigación que no se constriñen a lo material es uno de los principales aportes de los geógrafos culturales rupturistas. Después de la fiebre posmoderna comenzaron a surgir serias adver- tencias sobre la excesiva culturización de las nuevas geografías culturales, así como para mostrar que la cultura no es externa y forma parte íntegra de los procesos sociales (CLUA y ZUSMAN, 2002). Asimismo, un sector de los nuevos geógrafos culturales encabezado por PHILO (1999), manifestaba su preocupación por que la geografía podría perder su esencia y ser víctima de un fenómeno de des-materialización. Sin embargo, en la actualidad se observa como concurre una armonización entre lo tangible e intangible, abriendo nuevos caminos de investigación y superando las posiciones binarias.

Finalmente, las nuevas geografías culturales también fueron amonestadas por su carácter elitista y despolitizado. Argumento que se basó en la supresión de temáticas como la pobreza o la exclusión social en su campo de análisis. A pesar de estas críticas se observa que la geografía cultural es un campo fértil para el estudio, la denuncia y la crítica a las profundas desigualdades que yacen en el seno de las sociedades contemporáneas. Es así que la geografía es cada vez más diversa y abierta, lo cual debe celebrase más allá de los temores infundados a la desaparición de la esencia de la ciencia geográfica.

\section{Reflexiones finales}

El giro cultural y el surgimiento de las nuevas geografías culturales revitalizó el andamiaje teórico y metodológico de la Geografía Humana en un momento en el que cual se estaba replanteando la utilidad de la ciencia geográfica en el nuevo escenario global. Igualmente, ofreció nuevas maneras de pensar a la cultura y al espacio, que sin lugar a dudas abrieron un sitio a la ciencia geográfica en los debates de la teoría social en la cual el espacio y sus diferentes categorías tienen cada día mayor relevancia.

Los senderos de la geografía cultural no han sido lineales. Por el contrario, como se mostró en el documento se han manifestado cíclicos e incluso contradictorios. A veces enfatizando lo tangible, en otras ocasiones centrándose en lo inmaterial, aunque sin descartar que ambas dimensiones son necesarias y complementarias para ofrecer una comprensión profunda de la realidad social.

La geografía cultural se ha caracterizado por tener movimientos pendulares. Sus epicentros se han ubicado entre Europa y Norteamérica. En futras investigaciones podría explorarse la forma en que la geografía cultural ha sido recibida en el Sur Global, concretamente en Iberoamérica. Existe evidencia que la geografía en el Estado español experimentó una renovación a partir del intercambio entre geógrafas y geógrafos peninsulares con la geografía anglosajonas, como lo demuestra 
el liderazgo de Maria Dolors Garcia Ramon. Mientras que en América Latina existen trabajos innovadores de geografía cultural, aunque todavía habría que dimensionar sus impactos en el conjunto de la geografía. Sin lugar a dudas se tiene un panorama fértil por indagar.

\section{Referências}

BERDOULAY, V. (2012) Espaço e cultura. In: CASTRO I. et.al. (Orgs.). Olhares geográficos: modos de ver e viver o espaço. Rio de Janeiro: Bertrand Brasil, p.101-131.

CAPEL, H. (1981) Filosofía y ciencia en la geografía contemporánea. Barcelona: Barcanova.

CLAVAL, P. (2010) La geografía en recomposición: objetos que cambian, giros múltiples. ¿Disolución o profundización?". In: LINDÓN, A; Hiernaux, D. (Dirs.). Los giros de la geografía humana. Barcelona: Anthropos, p.63-82.

CLAVAL, P. (2003) A contribuição francesa ao desenvolvimento da abordagem cultural na geografía. In: LOBATO R.; ROSEDAHL, Z. (Orgs.), Introdução a geografia cultural. Rio de Janeiro: Bertrand Brasil, p. 147-166.

CLAVAL, P. (1999) Los fundamentos actuales de la geografía cultural. Documents d'Anàlisi Geogràfica, n. 34, 25-40.

CLAVAL, P. (1974) Evolución de la geografía humana. Barcelona: Oikos Tau.

CLUA, A. y P. ZUSMAN (2002). Más que palabras: otros mundos. Por una geografía cultural crítica. Documents d'Anàlisi Geogràfica, n. 34, 105-118.

COLLIGNON, B. (1999). La geografía cultural en Francia: un estado de la cuestión. Documents d'Anàlisi Geogràfica, n. 34, $103-117$.

CRANG, M. (1998). Cultural Geography. Londres: Routledge.

COSGROVE, D. (1983). Towards a radical cultural geography: problems of theory. Antipode, n. 15 (1), 1-11.

FERNÁNDEZ CHRISTLIEB, F. (2006) Geografía Cultural. In: HIERNAUX, D.; LINDÓN, A. (Dirs.). Tratado de Geografía Humana. Barcelona: Anthropos-Universidad Autónoma Metropolitana-Iztapalapa, pp.220-253.

GIMÉNEZ, G. (2007) Estudios sobre las culturas y las identidades sociales. México: Consejo Nacional para la Cultura y las Artes.

GÓMEZ MENDOZA, J., J. MUÑOZ y N. ORTEGA (1982) El pensamiento geográfico. Madrid: Alianza Editorial

JACKSON, P. (1999). ¿Nuevas geografías culturales? Documents d'Anàlisi Geogràfica, n. 34, 41-51.

JACKSON, P. (1980). A plea for cultural geography. Area n. 12, 110-113.

LINDÓN, A. (2016) Las geografías culturales de las afectividades ecarnadas. In: Lan, D. (Comp.). Geografías en diálogo. Tandil: UNCPBA. (pp. 9-24).

LINDÓN, A. (2010) Los giros teóricos: texto y contexto. In: LINDÓN, A; Hiernaux, D. (Dirs.). Los giros de la geografía humana. Barcelona: Anthropos, p.23-42.

LOBATO, R. (2001) Carl Sauer e a escola de Berkeley - uma apreciação. In: Rosendahl, Z.; Lobato, R. (Orgs.), Matrizes da geografia cultural. Rio de Janeiro: UERJ, p. 8-33.

LUNA, A. (1999). ¿Qué hay de nuevo en la nueva geografía cultural? Documents d'Anàlisi Geogràfica, n.34, 69-80.

MINCA, C. (2002). Más allá del posmodernismo. Viaje a través de la paradoja moderna. Documents d'Anàlisi Geogràfica, n. 40, 45-68.

NOGUÉ, J. y ABEL, A. (2007) Cartografía de los cambios sociales y culturales. In: ROMERO, J. (Coord.), Geografía humana: procesos, riesgos e incertidumbres en un mundo globalizado. Barcelona, Ariel, p. 159-197. 
ORTEGA VALCÁRCEL, J. (2000) Los horizontes de la geografía. Barcelona: Ariel.

PHILO, C. (1999). Más palabras, más mundos: reflexiones en torno al «giro cultural» y a la geografía social. Documents d'Anàlisi Geogràfica, n. 34, 81-99.

SAUER, C. (1925) (2006). La morfología del paisaje. Polis, n. 5 (15), 10-21.

KRAMSCH, O. (1999). El horizonte de la nueva geografía cultural. Documents d'Anàlisi Geogràfica, n. 34, 53-68.

ZUSMAN, P. y R. HAESBAERT (2011) Introducción. In: ZUSMAN, P; HAESBAERT, R.; CASTRO, H.; ADAMO, S.(Coords.), Geografías culturales: aproximaciones, intersecciones y desafíos. Buenos Aires: UBA: pp.5-18. 